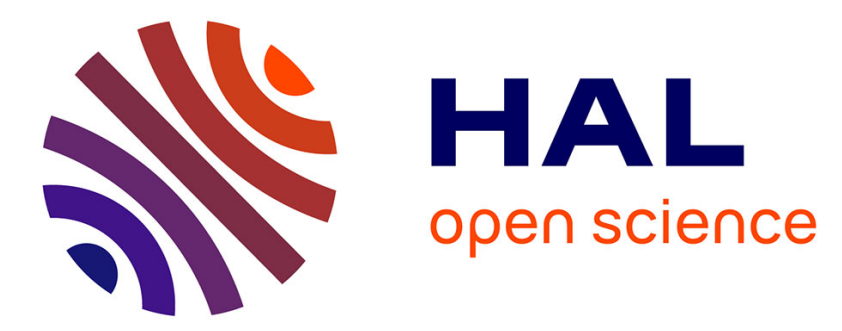

\title{
Visualization process of Temporal Data
}

Chaouki Daassi, Laurence Nigay, Marie-Christine Fauvet

\section{To cite this version:}

Chaouki Daassi, Laurence Nigay, Marie-Christine Fauvet. Visualization process of Temporal Data. Database and Expert Systems Applications (DEXA 2004), 2004, Zaragoza, Spain. pp.914-924. hal00953923

\section{HAL Id: hal-00953923 \\ https://hal.inria.fr/hal-00953923}

Submitted on 3 Mar 2014

HAL is a multi-disciplinary open access archive for the deposit and dissemination of scientific research documents, whether they are published or not. The documents may come from teaching and research institutions in France or abroad, or from public or private research centers.
L'archive ouverte pluridisciplinaire HAL, est destinée au dépôt et à la diffusion de documents scientifiques de niveau recherche, publiés ou non, émanant des établissements d'enseignement et de recherche français ou étrangers, des laboratoires publics ou privés. 


\title{
Visualization process of Temporal Data
}

\author{
Chaouki Daassi ${ }^{1}$, Laurence Nigay ${ }^{2}$ and Marie-Christine Fauvet ${ }^{2}$ \\ ${ }^{1}$ Laboratoire SysCom, Université de Savoie, Chambéry, France \\ ${ }^{2}$ Laboratoire CLIPS-IMAG BP 53-38041, Grenoble cedex 9, Grenoble, France \\ Chaouki.Daassi@univ-savoie.fr, [Laurence.Nigay, Marie-Christine.Fauvet]@imag.fr
}

\begin{abstract}
Temporal data are abundantly present in many application domains such as banking, financial, clinical, geographical applications and so on. Temporal data have been extensively studied from data mining and database perspectives. Complementary to these studies, our work focuses on the visualization techniques of temporal data: a wide range of visualization techniques have been designed to assist the users to visually analyze and manipulate temporal data. All the techniques have been designed independently. In such a context it is therefore difficult to systematically explore the set of possibilities as well as to thoroughly envision visualization techniques of temporal data. Addressing this problem, we present a visualization process of temporal data. We adapt the Ed Chi's visualization process to the case of temporal data. We illustrate the steps of our visualization process by considering the design of the Star Representation Technique that we have developed. By identifying and organizing the various aspects of design, our process serves as a basis for classifying existing visualization techniques and should also help the designer to address the right design questions and to envision future systems. Keywords: Visualization Technique, Visualization Process, Temporal Data.
\end{abstract}

\section{Introduction}

A temporal data denotes the evolution of an object characteristic over a period of time. The value of a temporal data is called a history. For the sake of simplicity, we define a history as a collection of instant time-stamped or interval time-stamped data items, although there are many other ways of representing a history [6]. Fig. 1, shows a history of numeric values (a monthly production of a firm), where each time-stamp denotes a month. The set of timestamps is the temporal domain of the observed data.

$[<400,01 / 1985>,<500,02 / 1985>,<450,03 / 1985>,<460,04 / 1985>,<480$, $05 / 1985>,<510,06 / 1985>,<600,07 / 1985>,<700,08 / 1985>,<720,09 / 1985>,<610$, $10 / 1985>\ldots \ldots \ldots<3240.01 / 2001><3250.02 / 2001>1$ 
Fig. 1. A collection of timestamped items.

Several visualization techniques of temporal data such as the ThemeRiver technique [9] have been proposed in the literature. In this paper, we present a visualization process for temporal data to structure the design space of these visualization techniques. We aim at defining the steps and their parameters that the designer should consider during the design. We adapt the Ed Chi's visualization process to the case of a temp oral data.

This paper is organized as follows: First (in Section 2), we briefly describe the Ed Chi's visualization process. In section 3, we show how Ed Chi's visualization process can be applied to temporal data. Finally, in section 4, we illustrate our visualization process by considering the design of the Star Representation Technique that we have developed.

\section{Ed Chi's visualization process}

As shown in Fig. 2, Ed Chi [4] decomposes the visualization process into three types of transformations manipulating four types of data. In this paper, we adopt the point of view of Vernier [12] that structures the visualization process of Ed Chi into four steps namely: (1) data, (2) point of view on the data, (3) visualization space and (4) point of view on the visualization space.

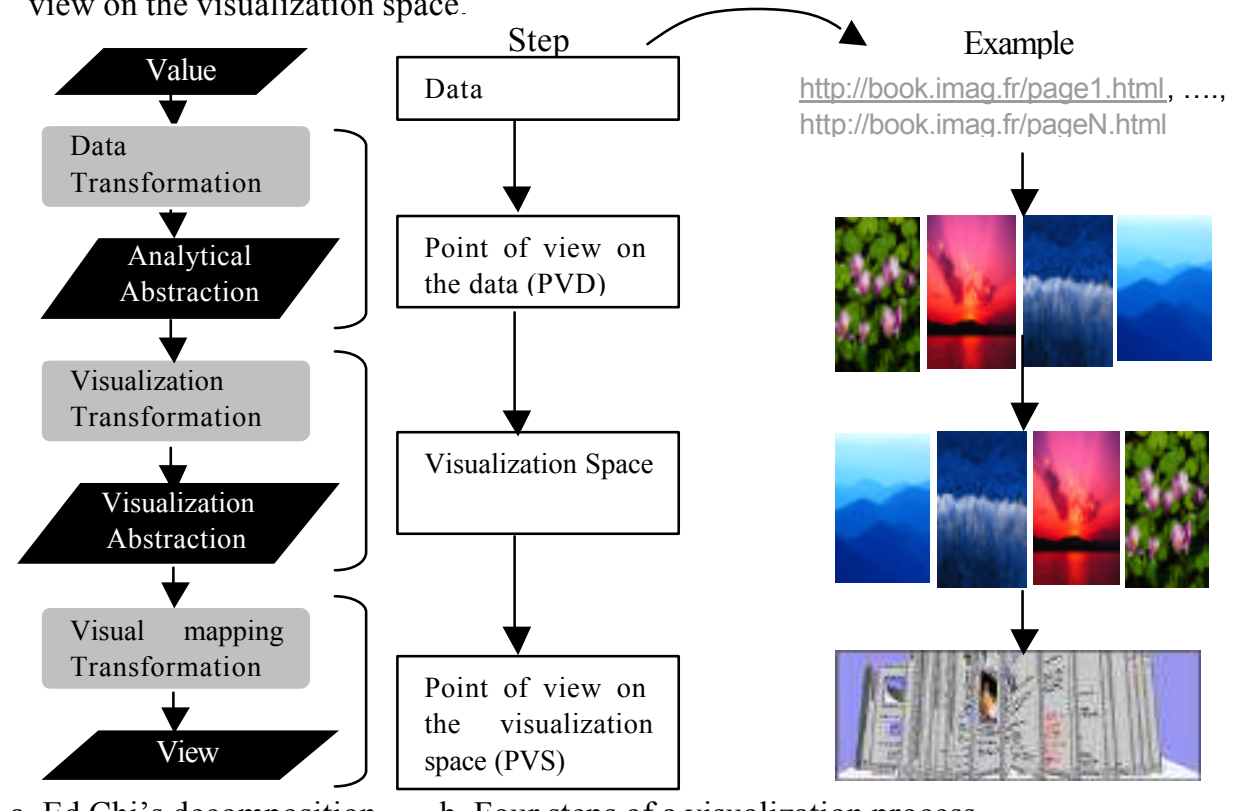

a. Ed Chi's decomposition

b. Four steps of a visualization process

Fig. 2. Ed Chi visualization process.

Data. This step is related to the data to be visualized. For example, as illustrated in Fig. 2, the data could be a set of URLs. 
Point of view on the data. At this stage of the process, the data are transformed onto an analytical representation. The point of view on the data is obtained by defining an analytical abstraction, in other words by obtaining meta-information, information related to the data such as their organization (hierarchical, sequential, etc.) or by filtering the initial space. For example, in Fig. 2 the web pages are retrieved from the list of URLs (initial data space), then an image is generated for each web page.

Visualization space. The visualization space is defined by a transformation of vis ualization which maps an analytical representation onto values ready to be displayed. In the visualization process shown in Fig. 2, the images (the point of view on the data) are ordered (ordered list of images). This transformation fixes the characteristics of the graphical space in which the data are visualized in terms of the number of its dimensions.

Point of view on the visualization space. The point of view on the visualization space produces the perceptible rendering. It results from a visual mapping transformation which maps the directly displayable values onto a graphical representation (view). In the example of Fig. 2, a book is defined for presenting the list of web pages as in [3]. At the previous step, the designer decides on the characteristics of the graphical space. During this step, the data values of the previous step are mapped onto graphical representations. In addition, at this step, a deformation function of the graphical space could be applied, for example a fisheye view for visualizing a huge amount of data.

In addition to the decomposition of the visualization process into three types of transformations manipulating four types of data, Ed Chi defines operators. We present some of these operators along the four steps of Fig. 2. Operators for the "data" step are for example data filtering, adding new sets of data and the Fourier transformation of images. Operators for the "point of view on the visualization space" step include rotation, translation, enlargement of graphical objects, and the positioning and orientation of a camera in a 3D scene. Other operators are defined for the two steps "point of view on data" and "visualization space".

Having presented the Chi's visualization process, we now apply this process to vis ualize temporal data.

\section{Visualization process of temporal data}

Fig. 3 distinguishes the two dimensions of a temporal data: structural dimension and temporal dimension. The structural dimension is characterized by a given data type. For example, we can distinguish one dimensional, two-dimensional and threedimensional data. One dimensional data could be either quantitative, nominal or ordered as defined by Card and Mackinlay [2]. Two dimensional data are for exa mple images or geographical maps. Three-dimensional data are for example real world objects.

Based on these two dimensions of a temporal data (i.e., structural and temporal dimensions), we define the visualization process as an association between two visualization 
processes dedicated to the two dimensions of the temporal data. The vis ualization process of the structural dimension is dependent on the type of data. Studying this process consists of studying the visualization process of data in general, which is out of the scope of this paper. We therefore only focus on the visualization process of the temporal dimension.

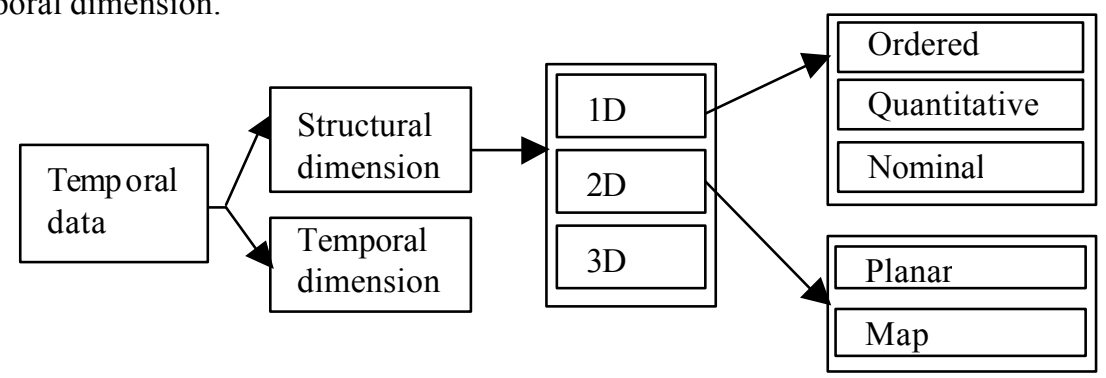

Fig. 3. Two dimensions of a temporal data.

Fig. 4 shows how we apply the Chi's visualization process to the temporal dimension of temporal data: four steps are defined namely time, point of view on time, time space and point of view on the time space.

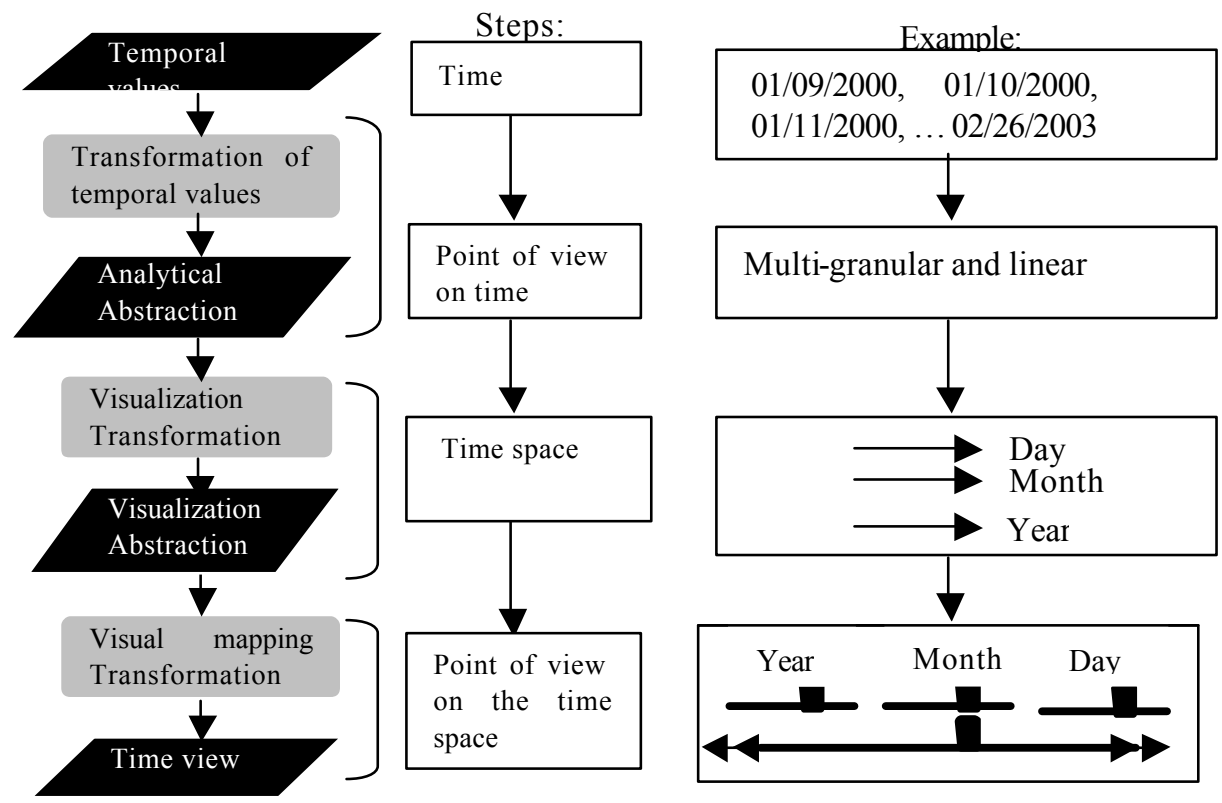

Fig. 4. Visualization process of a temporal dimension.

Time. This step represents the time values to be visualized. Time values are observed according to a time unit as Year, Month, Day, Hour, etc. In the example of Fig. 4, time values are observed according to the Day time unit.

Point of view on time. This step is defined by the transformation of the time values. This transformation extracts from the time values how time is represented: (i) in which 
unit system the time values are expressed (ii) are time values linear or periodic? In the example of Fig. 4, time is considered as multi-granular and linear. Multi-granular representation refers to time units composition: a year is composed of twelve months, a month is composed of 28, 29, 30 or 31 days, and so on.

Time space. At this step, displayable space of the time values are defined. For exa mple, time values observed at the unit Day (the analytical representation of "the point of view on time" step), could be mapped onto a matrix. One dimension of the matrix represents the months (Month is a unit coarser than Day), while the other represents the days. In the example of Fig. 4, each time unit is considered as linear: one time axis is then associated to each of them. Moreover at this step, the number of dimensions of the graphical representation in which the temporal values will be visualized is fixed. In the Cave Plots technique [1] of Fig. 5 the $\mathrm{X}$ axis is dedicated to represent time within a two-dimensional space. In this example, two representations of the time axis are used: one at the top and the other at the bottom of the representation. Other visualization techniques such as the Dynamic Timelines technique [10] represent time within a three-dimensional space.

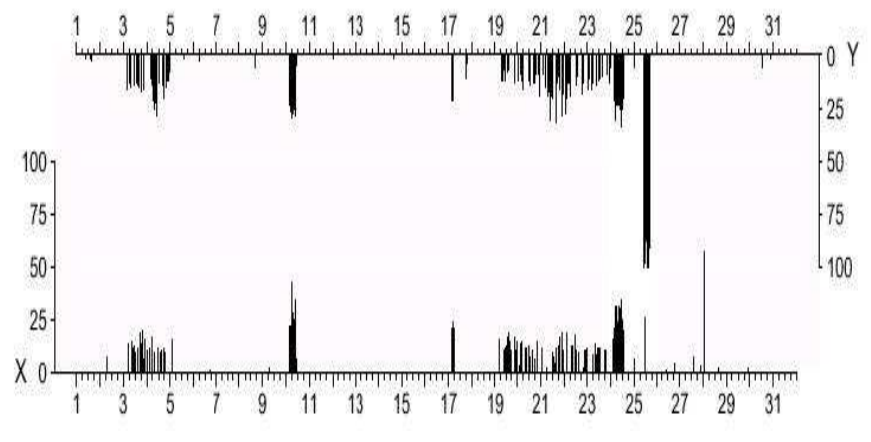

Fig. 5. Cave plots technique [1]

Point of view on the time space. The point of view on the time space refers to the perceptible rendering of the temporal dimension. At this step, the perceptible forms or representations of time are defined. These representations must be studied according to the human perception of time [8].

The choice of a representation is strongly influenced by the point of view on time that has been adopted (Step 2 "Point of view on time"). Indeed, the point of view on time identifies the characteristics of the temporal values to be visualized. For exa mple, a graphical representation of time in a cyclic form could be used because the temporal values are periodic: periodicity is a characteristic that has been identified during the step "Point of view on time". Fig. 6 shows a star representation to enhance a cyclic perception of time. Other representations such as the spiral representation in [13] could be used to enhance the cyclic perception of time. A linear representation is another classical way to express time, as depicted in Fig. 4. In the exa mple of Fig. 4, four timelines [7] are used to interact with the time space: one timeline for each time unit (Year, Month and Day) and one timeline to present an overview of the selected time values. 
In this section, we focused on the visualization process of the temporal dimension. However a temporal data is an association of two dimensions: a structural dimension and a temporal dimension. In the next section, we illustrate the complete visualization process of temporal data by considering the Star Representation Technique that we have developed. This visualization technique of temporal data relies on several forms of time used simultaneously making its design process challenging.

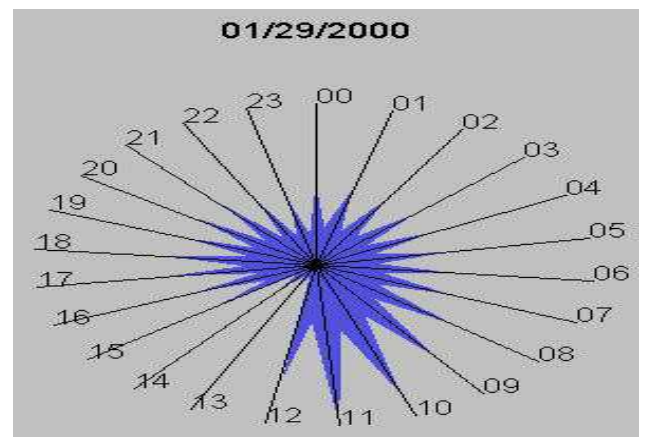

Fig. 6. Star representation [5].

\section{Star Representation Technique}

Fig. 7 presents the Star Representation Technique (SRT) while Fig. 8 presents its vis ualization process. As shown in Fig. 8, we distinguish two visualization processes: one dedicated to the temporal dimension (as described in the previous section) and one dedicated to the structural dimension.

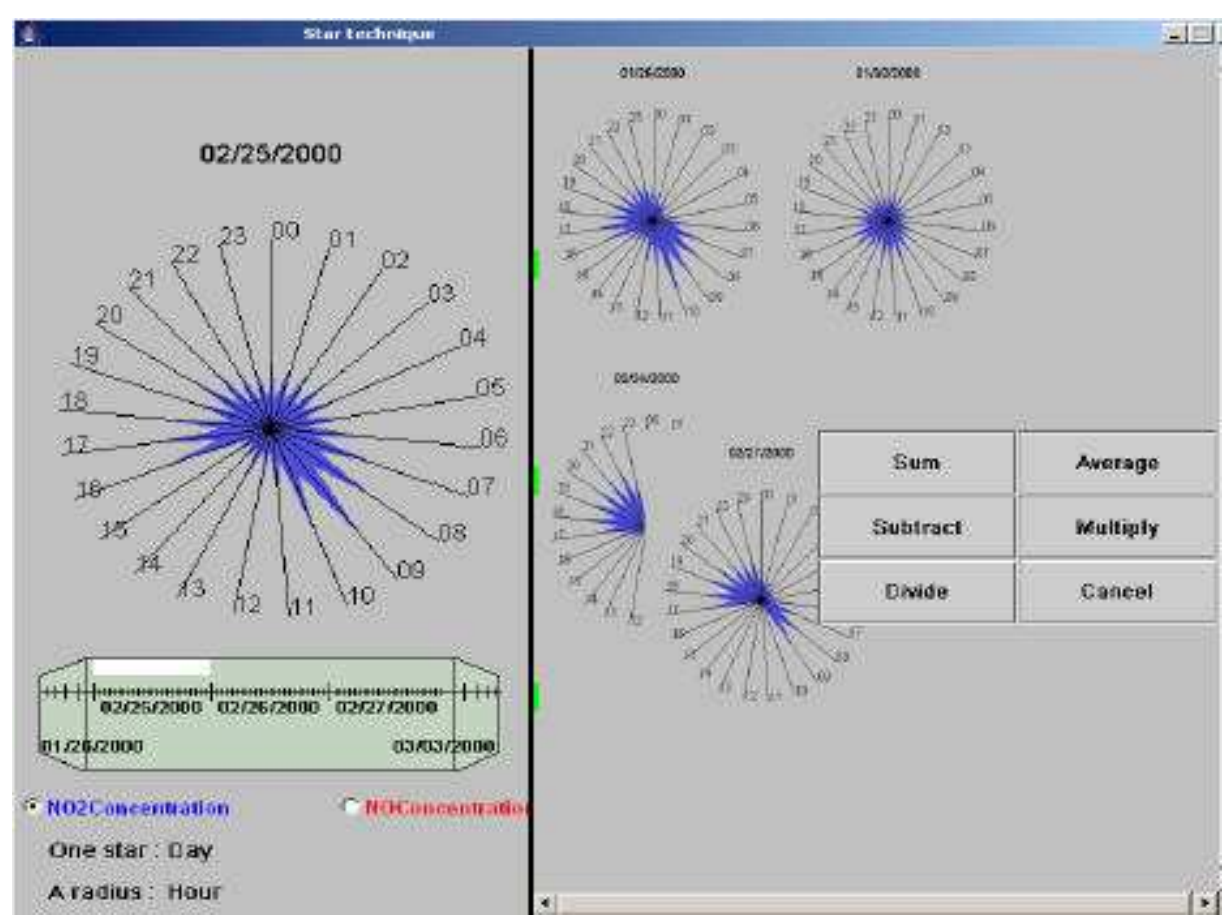


Fig. 7. Star representation technique.

The visualization process of the temporal dimension of the SRT (on the left in Fig. 8) is completely achieved independently from that of the structural dimension (on the right in Fig. 8), whereas the opposite is not no true. We note that other linking configurations between the two visualization processes exist: Considering the different linking configurations between the two visualization processes enables us to define classes of visualization techniques in [5].

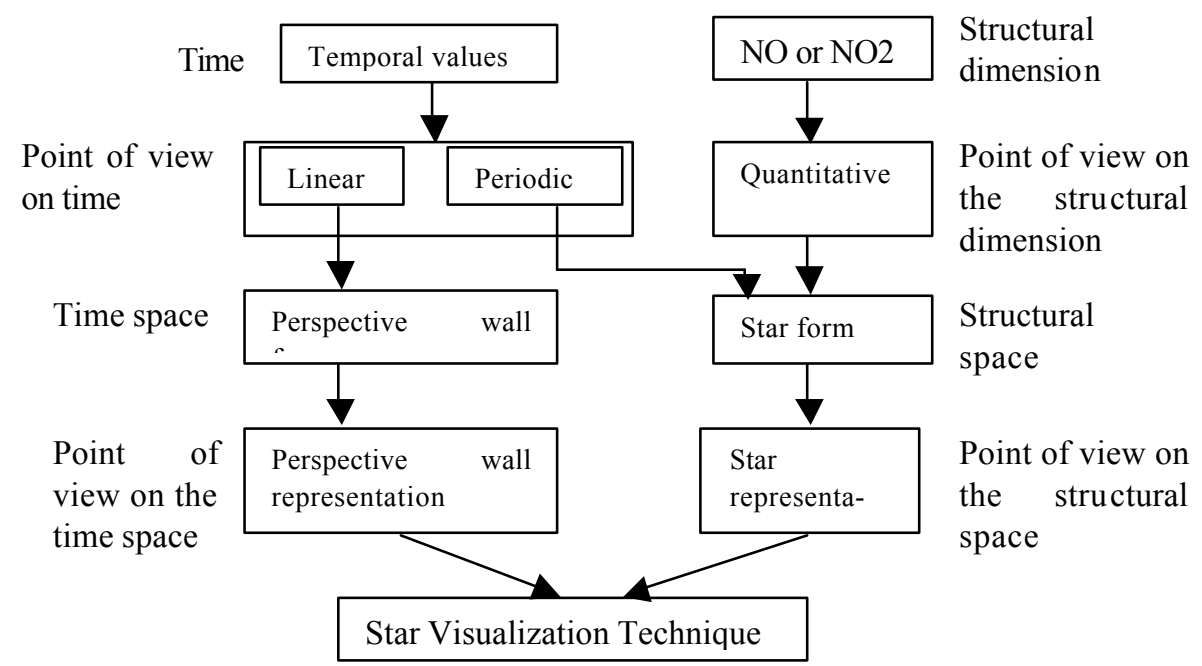

Fig. 8. Visualization process of Star Visualization Technique

As shown in Fig. 7, the SRT technique relies on two forms of time used simultaneously. In the visualization process of the temporal dimension of Fig. 8, two time perceptions are consequently considered at the point of view on time step: linear and periodic perceptions. The linear perception of time is used in the design of the perspective wall timeline, whereas the periodic perception is used in the design of the structural space, the star representation of Fig. 7.

\section{Perspective wall timeline}

We applied the visualization process (as described in the previous paragraph) to vis ualize the temporal dimension as a perspective wall timeline. As shown in Fig. 9, the perspective wall representation [11] distinguishes three facets: one in the front to represent data in detail, and two facets to represent the perspective effect. The perspective effect enhances the fact that time values are not limited to those visualized in 
the front of the timeline. The first and last observation instants of the data are visualized in the correspondent perspective facet (1921 and 1999 in Fig. 9). Consequently, the user can deduce the cardinality of the temporal domain.

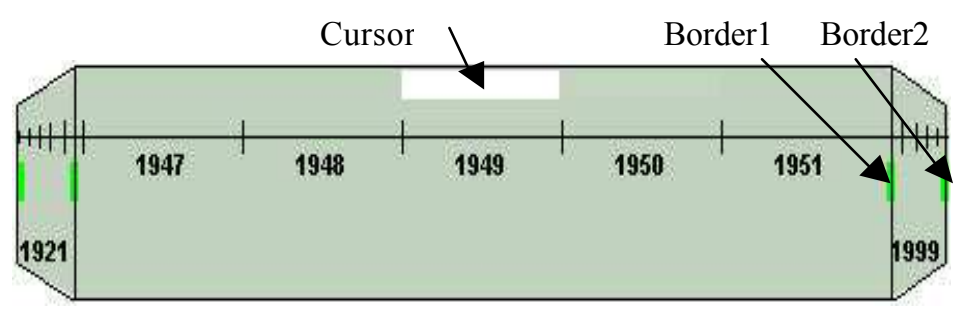

Fig. 9. Perspective wall timeline.

The user can customize the timeline: s/he can modify its size and the number of the visualized time values within its front facet. The form of a perspective wall provides two kinds of borders: Border1 of Fig. 9 delimits the facet in the front from the two perspective facets whereas Border2 delimits the timeline itself. By stretching the timeline from Border1, the user can augment or reduce the number of time values represented in the front facet. Similarly, by stretching the timeline from Border2, s/he can change the size of the timeline in order to improve the visibility of the vis ualized time values.

The perspective wall timeline offers two navigation modes to explore the time space. With the first mode, the user drags the cursor within the front facet of the timeline which leads to quick moving of time values. This mode is used when the user is searching a time value positioned far from the currently selected time value. By opposition, with the second mode, the user explores the time space step-by-step. To do so, $\mathrm{s} /$ he clicks in a perspective facet depending on the direction $\mathrm{s} /$ he wants.

As the visualization process of the temp oral dimension is realized independently from that of the structural dimension, the resulting time representation can be reused to implement several visualization techniques of temporal data. In addition to the SRT technique, we reused the perspective wall timeline in three different visualization techniques [5].

\section{Star Representation}

The visualization process of the structural dimension of the SRT is presented on the right in Fig. 8. During the visualization space step, a star representation is used to denote the evolution of the visualized history during a fixed-length period of time. The choice of the star representation is strongly influenced by the fact that we considered the periodic perception of time to define the visualization space (the anchoring point between the temporal and structural processes) and by the fact that the data are quantitative. In the example of Fig. 8, two quantitative temporal data are visualized, the NO 
and NO2 concentrations corresponding to pollution measures. In Fig. 7, a star denotes a day whereas the radii denotes the twenty four hours of a day.

The STR interface provides two visualization areas. The first one, located on the left in Fig. 7 and named the reference area, contains stars ordered in time. Within this area, a timeline is used to navigate through time. The displayed star corresponds to the period pointed by the cursor within the timeline. The user can directly observe the evolution of data over a given period (e.g. a year or a month) as well as from one period to the next. The second area located on the right in Fig. 7, the working area, enables the user to place selected stars in an arbitrary order. Putting stars close to each other facilitates the comparison of data values belonging to different periods. As shown in Fig. 7, when the user drags a star on top of another one, a palette of data manipulation operators appears. The user can for example compute the average of two temporal data then observe the result over time. The resulting data are represented as a star.

\section{Conclusion and future work}

We have presented a visualization process of temporal data. The goal of this work is to gain understanding of the design of visualization techniques of temporal data. The contribution of our visualization process is two-fold:

- First we structure the visualization process of a temporal data as an association of two visualization processes: one of the structural dimension and one of the temporal dimension. Several points of contact between the two processes are possible [5].

- Second we adapt the Ed Chi's visualization process to the case of temporal data.

We illustrated the visualization process by considering the design of the Star Representation Technique (SRT), one technique we have developed for interacting with temporal data. As the temporal dimension is designed independently from the structural dimension, our resulting perspective wall timeline has been reused in three other visualization techniques that are part of the INVEST system [5], a system that allows multiple views of the same temporal data. In a future work, we plan to use a dedicated physical device (as shown in Fig. 10) for the perspective wall timeline, making the reusable corresponding software component a reusable physical device. The visualization techniques such as the SRT will consequently be multi-surface, one for the temporal dimension and one for structural dimension.

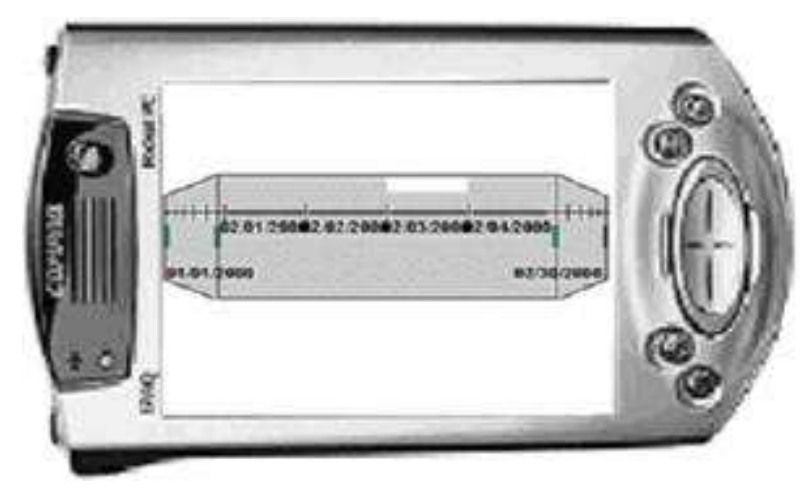


Fig. 10. Physical device to interact with the temporal dimension.

\section{References}

1. Becker R. A., Clark L. A. et Lambert D. Cave Plots: A Graphical Technique for Comparing Time Series. Journal of Computational and Graph. Stat., 3:3 (1994) 277283.

2. Card S. K. and Mackinlay Jock. The Structure on the Information Visualization Design Space. In Proc. of InfoVis (1997).

3. Card S. K., Robertson G. G., York W. The WebBook and the Web Forager: An Information Workspace for the World-Wide Web (1996). In Proc. of the Conference on Human Factors in Computing Systems CHI'96.

4. Chi E. H. A Taxonomy of Visualization Techniques using the Data State Reference Model. In Proc. of InfoVis (2000).

5. Daassi C. Techniques d'interaction avec un espace de données temporelles. $\mathrm{PhD}$ thesis in Computer Science from Joseph-Fourier University of Grenoble, France, 2003. Available at http://iihm.imag.fr/publs/2003/theseChaoukiDaassi.pdf

6. Dumas M, Tempos: une plate-forme pour le développement d'application temporelles au dessus de SGBD à objets. $\mathrm{PhD}$ thesis in Computer Science from JosephFourier University of Grenoble, France, (2000).

7. Dumas M., Daassi C., Fauvet M-C, Nigay N. "Pointwise Temporal Object Database Browsing". In Proc. Of ECOOP, France, (2000) 170-184.

8. Freeman W. J. Perception of Time and Causation through the Kinesthesia of intentional action. Cognitive Processing 1, In International Quarterly of Cognitive Science, 18-34.

9. Havre S., Hetzler B. and Nowell L. ThemeRiver: Visualizing Theme changes over Time. In Proc. of the IEEE Symposium on Information Visualization 2000.

10. Kullberg R. L. Dynamic Timelines: Visualizing Historical Information in Three Dimensions. Master Thesis from Media Lab. MIT, (1995).

11. Mackinlay J.D., Robertson G., and Card S.K. The Perspective wall: Detail and Context smoothly integrated, Human factors in computing systems conference proceedings on Reaching through technology, (1991) 173 - 176.

12. Vernier F. La multimodalité en sortie et son application à la visualisation de grandes quantités d'information. PhD thesis in Computer Science from Joseph-Fourier University of Grenoble, France, (2001).

13. Weber M., Alexa M. et Müller W. Visualizing Time-Series on Spirals. In Proc. of InfoVis (2001). 